IISc-CHEP/01/09

IMSC-2009/01/01

SU-4252-883

\title{
Spontaneous Symmetry Breaking In Twisted Noncommutative Quantum Theories
}

\author{
A. P. Balachandran* \\ Cátedra de Excelencia, Departamento de Matemática, \\ Universidad Carlos III de Madrid, \\ 28911, Leganés, Madrid, Spain \\ and \\ Physics Department, Syracuse University Syracuse, \\ $N Y, 13244-1130, U S A$ \\ T. R. Govindarajan ${ }^{\dagger}$ \\ The Institute of Mathematical Sciences C. I. T. Campus Taramani, Chennai 600 113, India \\ Sachindeo Vaidya ${ }^{\ddagger}$ \\ Centre for High Energy Physics, Indian Institute of Science, Bangalore 560 012, India
}

\begin{abstract}
We analyse aspects of symmetry breaking for Moyal spacetimes within a quantisation scheme which preserves the twisted Poincaré symmetry. Towards this purpose, we develop the LSZ approach for Moyal spacetimes. The latter gives a formula for scattering amplitudes on these spacetimes which can be obtained from the corresponding ones on the commutative spacetime. This formula applies in the presence of spontaneous breakdown of symmetries as well. We also derive Goldstone's theorem on Moyal spacetime. The formalism developed here can be directly applied to the twisted standard model.
\end{abstract}

PACS numbers: $11.10 . \mathrm{Nx}, 11.30 . \mathrm{Cp}$

Keywords: Gauge fields, Non-Commutative Geometry, twisted Poincaré symmetry

\section{INTRODUCTION}

Spontaneous breaking of a continuous symmetry involves a subtle interplay between an infinite number of degrees of freedom, local and spacetime symmetries, dimension of spacetime, and the notion of (non-)locality of interaction. Naturally one would suspect that the phenomenon of spontaneous symmetry breaking (SSB) leads to different physics in the context of quantum field theories on the Groenewold-Moyal (GM) plane, when the idea of locality is altered, albeit in a very precise sense: pointwise multiplication of two functions is replaced by star-multiplication, which is no longer commutative, and is in addition non-local. New phases and soliton solutions appear making the dynamics richer [1, 2, 3, 4].

Writing quantum field theories on such spaces requires some care, if one wishes to discuss questions related to Poincaré invariance. To give up this spacetime symmetry almost entirely (which is what conventional quantization does) seems too heavy a price, as it affects the notion of identity of particles ("of two identical particles in one frame should describe two identical particles in all reference frames"), and leads to unacceptable coupling between UV- and IR- degrees of freedom as well [5]. The program of twisted quantization initiated in [6, 7] on the the other hand, avoids these pitfalls: Poincaré invariance can be maintained, a generalized notion of identical particles can be defined, and UV and IR degrees of freedom decouple nicely [8], thus rekindling the hope that phenomenologically interesting models can be constructed. Indeed one can construct quantum gauge theories with arbitrary gauge groups consistently $[9]$.

In this paper, we address the issue of SSB and Higgs phenomenon in twisted quantum theories, and demonstrate signatures of noncommutativity. Our general formulation applies to an arbitrary group $G$ breaking to a subgroup

\footnotetext{
*bal@phy.syr.edu

$\dagger$ trg@imsc.res.in

¥ vaidya@cts.iisc.ernet.in
} 
$H$. The extension to the (noncommutative) Standard Model and beyond-Standard Model physics is conceptually straightforward, and will be discussed as well. Such physics merits a more elaborate investigation which we reserve for later work.

This paper is organised as follows. In Section 2, we will review twisted quantization on noncommutative spaces and gauge theories based on this formalism. Section 3 will elaborate the LSZ formalism for twisted quantisation and discuss in detail the Gell-Mann-Low formula and its modifications on the GM plane. In Section 4, we will summarise our rules for twisted quantum field theories followed by application of the same to spontaneously broken theories and Higgs mechanism on the GM plane in Sec.5. Our conclusions and future outlook are in Section 6.

\section{TWISTED QUANTIZATION}

The algebra of functions $\mathcal{A}_{\theta}\left(\mathbb{R}^{N}\right)$ on the GM plane consists of smooth functions on $\mathbb{R}^{N}$, with the multiplication map

$$
\begin{aligned}
m_{\theta}: \mathcal{A}_{\theta}\left(\mathbb{R}^{N}\right) \otimes \mathcal{A}_{\theta}\left(\mathbb{R}^{N}\right) & \rightarrow \mathcal{A}_{\theta}\left(\mathbb{R}^{N}\right) \\
\alpha \otimes \beta & \rightarrow \alpha e^{\frac{i}{2} \overleftarrow{\partial}_{\mu} \theta^{\mu \nu} \vec{\partial}_{\nu} \beta:=\alpha * \beta}
\end{aligned}
$$

where $\theta^{\mu \nu}$ is a constant antisymmetric tensor.

Let

$$
F_{\theta}=e^{\frac{i}{2} \partial_{\mu} \otimes \theta^{\mu \nu} \partial_{\nu}}=\text { "Twist element". }
$$

Then

$$
m_{\theta}(\alpha \otimes \beta)=m_{0}\left[F_{\theta} \alpha \otimes \beta\right]
$$

where $m_{0}$ is the point-wise multiplication map, also defined by (11).

The usual action of the Lorentz group $\mathcal{L}$ is not compatible with $*$-multiplication: transforming $\alpha$ and $\beta$ separately by an arbitrary group element $\Lambda \in \mathcal{L}$ and then $*$-multiplying them is not the same as transforming their $*$-product. In other words, the usual coproduct $\Delta_{0}(\Lambda)=\Lambda \otimes \Lambda$ on the group algebra $\mathbb{C} \mathcal{L}$ of $\mathcal{L}$ is not compatible with $m_{\theta}$. But a new coproduct $\Delta_{\theta}$ obtained using the twist is compatible, where

$$
\Delta_{\theta}(\Lambda)=F_{\theta}^{-1} \Delta_{0}(\Lambda) F_{\theta}
$$

Here $\partial_{\mu} \otimes \theta^{\mu \nu} \partial_{\nu}$ in $F_{\theta}$ is to be replaced by $\quad-P_{\mu} \otimes \theta^{\mu \nu} P_{\nu}$ where $P_{\mu}$ are translation generators: we are dealing with $\mathcal{P}_{\theta} \otimes \mathcal{P}_{\theta}$ where $\mathcal{P}_{\theta}$ is a Hopf algebra associated with the Poincaré group algebra $\mathbb{C} \mathcal{P}$ with the coproduct (4).

This twisted coproduct does not preserve standard (anti-)symmetrization, because it does not commute with the usual flip operator $\tau_{0}$ defined by $\tau_{0}:(\alpha \otimes \beta)=(\beta \otimes \alpha)$ :

$$
\Delta_{\theta}(\Lambda) \tau_{0} \neq \tau_{0} \Delta_{\theta}(\Lambda)
$$

On the other hand, it does preserve twisted (anti-)symmetrization, defined using a new flip operator $\tau_{\theta}=$ $F_{\theta}^{-1} \tau_{0}(\Lambda) F_{\theta}$ :

$$
\Delta_{\theta}(\Lambda) \tau_{\theta}=\tau_{\theta} \Delta_{\theta}(\Lambda)
$$

Thus in noncommutative quantum theory, the usual fermions/bosons do not make sense, but twisted ones do. They are obtained from the projectors $S_{\theta}, A_{\theta}$ :

$$
S_{\theta}=\frac{\mathbf{1}+\tau_{\theta}}{2}, \quad A_{\theta}=\frac{\mathbf{1}-\tau_{\theta}}{2} .
$$

\section{A. Quantum Fields}

A quantum field $\chi$ on evaluation at a spacetime point (or more generally on pairing with a test function) gives an operator acting on a Hilbert space. A field at $x_{1}$ acting on the vacuum gives a one-particle state centered at $x_{1}$. When we write $\chi\left(x_{1}\right) \chi\left(x_{2}\right)$ we mean $(\chi \otimes \chi)\left(x_{1}, x_{2}\right)$. Acting on the vacuum we generate a two-particle state, where one particle is centered at $x_{1}$ and the other at $x_{2}$. 
If $a_{p}$ is the annihilation operator of the free second-quantized field $\phi_{\theta}$ on $\mathcal{A}_{\theta}\left(\mathbb{R}^{N}\right)$, we want, as in standard quantum field theory,

$$
\begin{aligned}
\left\langle 0\left|\phi_{\theta}(x) a_{p}^{\dagger}\right| 0\right\rangle & =e_{p}(x), \\
\frac{1}{2}\left\langle 0\left|\phi_{\theta}\left(x_{1}\right) \phi_{\theta}\left(x_{2}\right) a_{q}^{\dagger} a_{p}^{\dagger}\right| 0\right\rangle & =\left(\frac{\mathbf{1} \pm \tau_{\theta}}{2}\right)\left(e_{p} \otimes e_{q}\right)\left(x_{1}, x_{2}\right) \\
& \equiv\left(e_{p} \otimes_{S_{\theta}, A_{\theta}} e_{q}\right)\left(x_{1}, x_{2}\right)
\end{aligned}
$$

where $e_{p}(x)=e^{-i p \cdot x}$.

This compatibility between twisted statistics and Poincaré invariance has profound consequences for commutation relations. For example when the states are labeled by momenta, we have, from exchanging $p$ and $q$ in (9),

$$
|p, q\rangle_{S_{\theta}, A_{\theta}}= \pm e^{i \theta^{\mu \nu} p_{\mu} q_{\nu}}|q, p\rangle_{S_{\theta}, A_{\theta}} .
$$

This is the origin of the commutation relations

$$
\begin{aligned}
a_{p}^{\dagger} a_{q}^{\dagger} & = \pm e^{i \theta^{\mu \nu} p_{\mu} q_{\nu}} a_{q}^{\dagger} a_{p}^{\dagger} \\
a_{p} a_{q} & = \pm e^{i \theta^{\mu \nu} p_{\mu} q_{\nu}} a_{q} a_{p} .
\end{aligned}
$$

\section{B. Gauge Theories}

The algebra $\mathcal{A}_{\theta}\left(\mathbb{R}^{N}\right)$, regarded as a vector space, is a module for $\mathcal{A}_{0}\left(\mathbb{R}^{N}\right)$. This observation is of central importance to us, as it allows us to write gauge theories based on arbitrary gauge groups (as opposed to just $U(N)$ ). We can show this as follows.

For any $\alpha \in \mathcal{A}_{\theta}\left(\mathbb{R}^{N}\right)$, we can define two representations $\hat{\alpha}^{L, R}$ acting on $\mathcal{A}_{\theta}\left(\mathbb{R}^{N}\right)$ :

$$
\hat{\alpha}^{L} \xi=\alpha * \xi, \quad \hat{\alpha}^{R} \xi=\xi * \alpha \quad \text { for } \quad \xi \in \mathcal{A}_{\theta}\left(\mathbb{R}^{N}\right),
$$

where $*$ is the GM product defined by Eq.(11) (or, equivalently, by Eq.(3)). The maps $\alpha \rightarrow \hat{\alpha}^{L, R}$ have the properties

$$
\begin{aligned}
\hat{\alpha}^{L} \hat{\beta}^{L} & =(\hat{\alpha} \hat{\beta})^{L}, \\
\hat{\alpha}^{R} \hat{\beta}^{R} & =(\hat{\beta} \hat{\alpha})^{R}, \\
{\left[\hat{\alpha}^{L}, \hat{\beta}^{R}\right] } & =0 .
\end{aligned}
$$

The reversal of $\hat{\alpha}, \hat{\beta}$ on the right-hand side of (15) means that for position operators,

$$
\left[\hat{x}^{\mu L}, \hat{x}^{\nu L}\right]=i \theta^{\mu \nu}=-\left[\hat{x}^{\mu R}, \hat{x}^{\nu R}\right] .
$$

Hence in view of (16),

$$
\hat{x}^{\mu c}=\frac{1}{2}\left(\hat{x}^{\mu L}+\hat{x}^{\mu R}\right)
$$

generates a representation of the commutative algebra $\mathcal{A}_{0}\left(\mathbb{R}^{N}\right)$ :

$$
\left[\hat{x}^{\mu c}, \hat{x}^{\nu c}\right]=0 .
$$

We regard elements of the gauge group $\mathcal{G}$ as maps from $\mathcal{A}_{0}\left(\mathbb{R}^{N}\right)$ to the Lie group $G$.

$$
g: \hat{x^{c}} \longrightarrow g\left(\hat{x^{c}}\right) \in G
$$

In cases of interest, $G$ is a compact connected Lie group . For convenience, we also think of $G$ concretely in terms of the defining finite-dimensional faithful representation by linear operators.

Fields which transform non-trivially under $\mathcal{G}$ are modules over $\mathcal{A}_{\theta}\left(\mathbb{R}^{N}\right)$. If a $d$-dimensional representation of $G$ is involved, they can be elements of $\mathcal{A}_{\theta}\left(\mathbb{R}^{N}\right) \otimes \mathbb{C}^{d}$. Compatibility of gauge transformations (on these modules) with the *-product requires us to twist the coproduct on the gauge group too. The new coproduct is

$$
\Delta_{\theta}\left(g\left(\hat{x}^{c}\right)\right)=F_{\theta}^{-1}\left[g\left(\hat{x}^{c}\right) \otimes g\left(\hat{x}^{c}\right)\right] F_{\theta} .
$$


This deformation of the coproduct for gauge transformations is neccessary if we want to be able to construct gauge scalars, and other composite operators (see [9] for details).

Finally, we need to understand how to define covariant derivative $D_{\mu}$. To this end, consider for simplicity a free charged scalar field $\phi_{\theta}(x)$,

$$
\phi_{\theta}(x)=\int d \mu(p)\left(a_{p} e^{-i p \cdot x}+b_{p}^{\dagger} e^{i p \cdot x}\right), \quad \text { where } \quad d \mu(p) \equiv \frac{d^{3} p}{2 p_{0}}, \quad p_{0}=\sqrt{\vec{p}^{2}+m^{2}}
$$

We require that the field $\phi_{\theta}$ obeys twisted statistics in Fock space:

$$
\begin{aligned}
& a_{p} a_{q}=e^{i p \wedge q} a_{q} a_{p}, \quad \text { where } p \wedge q \equiv p_{\mu} \theta^{\mu \nu} q_{\nu}, \\
& a_{p} a_{q}^{\dagger}=e^{-i p \wedge q} a_{q}^{\dagger} a_{p}+2 p_{0} \delta^{(3)}(p-q)
\end{aligned}
$$

and similarly for $b(p), b^{\dagger}(p)$. The twisted operators $a(p), a^{\dagger}(p), b(p), b^{\dagger}(p)$ can be realized in terms of untwisted Fock space operators $c(p), d(p)$ and their adjoints through the well-known "dressing transformations" 10]

$$
\begin{aligned}
a_{p} & =c_{p} e^{-\frac{i}{2} p \wedge P}, \\
b_{p} & =d_{p} e^{-\frac{i}{2} p \wedge P}, \quad a_{p}^{\dagger}=c_{p}^{\dagger} e^{\frac{i}{2} p \wedge P}, \\
P_{\mu} & =\int d \mu(p) p_{\mu}\left(a_{p}^{\dagger} a_{p}+b_{p}^{\dagger} b_{p}\right)=\int d \mu(p) p_{\mu} e^{\frac{i}{2} p \wedge P}, \text { where } \\
& =\text { total momentum operator. }
\end{aligned}
$$

Then $\phi_{\theta}(x)$ may be written in terms of the ordinary or commutative fields $\phi_{0}$ as

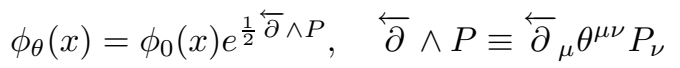

As $\mathcal{A}_{\theta}\left(\mathbb{R}^{N}\right)$ is a module for $\mathcal{A}_{0}\left(\mathbb{R}^{N}\right)$, we require that the covariant derivative respects this property. In addition, we also require that in quantum theory, the covariant derivative preserves statistics, and also Poincaré and gauge symmetries. The only possibility that satisfies these requirements is

$$
D_{\mu} \phi_{\theta}=\left(\left(D_{\mu}\right)_{0} \phi_{0}\right) e^{\frac{1}{2} \overleftarrow{\partial} \wedge P}
$$

where $\left(D_{\mu}\right)_{0}=\partial_{\mu}+\left(A_{\mu}\right)_{0}$ and $\left(A_{\mu}\right)_{0}$ is the commutative gauge field, depending on $\hat{x}^{c}$ only.

The commutator of two covariant derivatives gives us the curvature:

$$
\left[D_{\mu}, D_{\nu}\right] \phi=\left(\left[\left(D_{\mu}\right)_{0},\left(D_{\nu}\right)_{0}\right]\right) e^{\frac{1}{2} \overleftarrow{\partial} \wedge P}=\left[\left(F_{\mu \nu}\right)_{0} \phi_{0}\right] e^{\frac{1}{2} \overleftarrow{\partial} \wedge P}
$$

The field strength $\left(F_{\mu \nu}\right)_{0}$ transforms correctly (i.e. covariantly) under gauge transformations, so we can use it to construct the Hamiltonian of the quantum gauge theory. Pure gauge theories on the GM plane are thus identical to their commutative counterparts.

Below we will outline an approach to scattering theory of twisted fields based on the LSZ formalism. In that approach, (26) and (27) are true in the fully interacting case as well. Thus these equations are valid with $P_{\mu}$ being the total four momentum of all fields including interactions.

We will discuss spontaneous symmetry breakdown in the presence of twists later. Here we remark only the following. If the connected, compact Lie group $G$ is the gauged group and it is spontaneously broken to the gauge theory of the subgroup $H$, then the vector fields acquiring mass are to be twisted. Only the gauge fields of $H$ escape the twist.

The LSZ approach to the scattering theory of such interactions appears to be more streamlined and non-perturbative as compared to our earlier treatments [8]. There we used the interaction representation. In this representation, the free Hamiltonian is not twisted whereas the interaction Hamiltonian is

$$
H_{\theta}^{I}=\int d^{3} x\left[\mathcal{H}_{\theta}^{M-G}+\mathcal{H}_{\theta}^{G}\right]
$$

Here $\mathcal{H}_{\theta}^{M-G}$ and $\mathcal{H}_{\theta}^{G}$ correspond to the interaction Hamiltonian densities with matter and gauge fields, and with gauge fields alone respectively. The $\theta$ - dependence of the interaction representation S-matrix disappears when $\mathcal{H}_{\theta}^{G}=0$, but that is not the case when both $\mathcal{H}_{\theta}^{M}$ and $\mathcal{H}_{\theta}^{G}$ are present. Scattering processes that involve cross terms between $\mathcal{H}_{\theta}^{M-G}$ and $\mathcal{H}_{\theta}^{G}$, like the $q g \rightarrow q g$ scattering in QCD, show effects of noncommutativity.

As we will later point out, for reasons not well understood, for $\theta^{\mu \nu} \neq 0$, the LSZ S-matrix differs from the interaction representation S-matrix and leads to different cross sections. 


\section{THE LSZ FORMALISM FOR TWISTED QUANTUM THEORIES}

In the next two subsections below, we review scattering theory, including the LSZ formalism for standard (untwisted fields). We then generalise the discussion to the twisted case.

\section{A. Formal scattering theory}

In standard scattering theory, the Hamiltonian $H$ is split into a "free" Hamiltonian $H_{0}$ and an "interaction" piece $H_{I}$,

$$
H=H_{0}+H_{I}
$$

and $H_{0}$ is used to define the states in the infinite past and future. Then the states at $t=0$ which in the infinite past (future) become states evolving by $H_{0}$ are the in (out) states:

$$
\begin{aligned}
&\left.e^{-i H T_{-}} \mid \psi ; \text { in }\right\rangle \stackrel{T_{-} \rightarrow-\infty}{\longrightarrow} e^{-i H_{0} T_{-}}|\psi ; \mathrm{F}\rangle, \quad \mathrm{F} \equiv \text { free } \\
&\left.e^{-i H T_{+}} \mid \psi ; \text { out }\right\rangle \stackrel{T_{+} \rightarrow+\infty}{\longrightarrow} e^{-i H_{0} T_{+}}|\psi ; \mathrm{F}\rangle .
\end{aligned}
$$

Hence

$$
\begin{aligned}
\mid \psi ; \text { in }\rangle & =\Omega_{+}|\psi ; \mathrm{F}\rangle \\
\mid \psi ; \text { out }\rangle & =\Omega_{-}|\psi ; \mathrm{F}\rangle, \\
\Omega_{ \pm} & \equiv e^{i H T_{\mp}} e^{-i H_{0} T_{\mp}}, \quad \text { as } \quad T_{\mp} \rightarrow \mp \infty \\
& =\mathrm{M} \phi \text { ller operators. }
\end{aligned}
$$

We see that

$$
\begin{aligned}
e^{i H \tau} \Omega_{ \pm} & =\lim _{T_{\mp} \rightarrow \mp \infty} e^{i H T_{\mp}} e^{-i H_{0}\left(T_{\mp}-\tau\right)}, \\
& =\Omega_{ \pm} e^{i H_{0} \tau}
\end{aligned}
$$

and

$$
\left.\mid \psi ; \text { out }\rangle=\Omega_{-} \Omega_{+}^{\dagger} \mid \psi ; \text { in }\right\rangle
$$

If the incoming state is $\left|k_{1}, k_{2}, \cdots k_{N} ; \mathrm{F}\right\rangle$, it follows that

$$
\left.\mid k_{1}, k_{2}, \cdots k_{N} ; \text { in }\right\rangle=\Omega_{+}\left|k_{1}, k_{2}, \cdots k_{N} ; \mathrm{F}\right\rangle
$$

has eigenvalue $\sum k_{i 0}$ for the total Hamiltonian $H$. A similar result is true for

$$
\left.\mid k_{1}, k_{2}, \cdots k_{N} ; \text { out }\right\rangle=\Omega_{-}\left|k_{1}, k_{2}, \cdots k_{N} ; \mathrm{F}\right\rangle .
$$

We note that the scattering amplitude is

$$
\langle\xi ; \text { out }| \psi ; \text { in }\rangle=\left\langle\xi ; \text { in }\left|\Omega_{+} \Omega_{-}^{\dagger}\right| \psi ; \text { in }\right\rangle .
$$

In other words, the LSZ $S$-matrix is

$$
\left.\left.S=\Omega_{+} \Omega_{-}^{\dagger}, \quad \mid \psi ; \text { out }\right\rangle=S^{\dagger} \mid \psi ; \text { in }\right\rangle .
$$

Between the "free" states, the $S$-operator is different:

$$
\langle\xi ; \text { out }| \psi ; \text { in }\rangle=\left\langle\xi ; \mathrm{F}\left|\Omega_{-}^{\dagger} \Omega_{+}\right| \psi ; \mathrm{F}\right\rangle .
$$

The LSZ formalism works exclusively with the in- and out-states, as Haag's theorem shows that $\Omega_{ \pm}$do not ex-

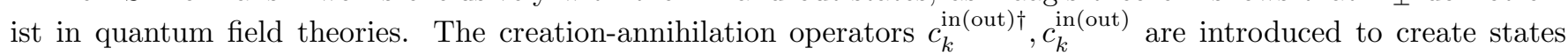


$\mid k_{1}, k_{2}, \cdots k_{N} ;$ in(out) $\rangle$ from the vacuum. The in- and out- fields $\phi_{\text {in(out) }}$ are then defined using superposition. They look like free fields, but are not, since for the total four-momentum $P_{\mu}$, we have

$$
\left.\left.P_{\mu} \mid k_{1}, k_{2}, \cdots k_{N} ; \operatorname{in}(\text { out })\right\rangle=\left(\sum_{i} k_{i \mu}\right) \mid k_{1}, k_{2}, \cdots k_{N} ; \text { in }(\text { out })\right\rangle .
$$

It is also assumed that

(a) The vacuum and single particle states are unique up to a phase. Then after a phase choice, there is only one vacuum $|0\rangle,\langle 0 \mid 0\rangle=1$, and

$$
S|0\rangle=|0\rangle .
$$

(b) There exists an interpolating field $\phi$ in the Heisenberg representation such that matrix elements of $\phi(x, t)$ between in- and out- states go to those of $\phi_{\text {in,out }}(x, t)$ in the infinite past and future,

$$
\phi(x, t)-\phi_{\text {in }, \text { out }}(x, t) \rightarrow 0 \quad \text { as } \quad t \rightarrow \mp \infty
$$

in weak topology. (We treat the case of just one scalar field for simplicity.)

Then LSZ formalism shows that

$$
\left.\left\langle k_{1}^{\prime}, k_{2}^{\prime}, \cdots k_{N}^{\prime} ; \text { out }\right| k_{1}, k_{2}, \cdots k_{M} ; \text { in }\right\rangle=\int \mathcal{I} G_{N+M}\left(x_{1}^{\prime}, \cdots, x_{N}^{\prime} ; x_{1} \cdots x_{M}\right)
$$

where

$$
\mathcal{I}=\prod d^{4} x_{i}^{\prime} \prod d^{4} x_{j} e^{i\left(k_{i}^{\prime} \cdot x_{i}^{\prime}-k_{j} \cdot x_{j}\right)} i\left(\partial_{i}^{\prime 2}+m^{2}\right) \cdot i\left(\partial_{j}^{2}+m^{2}\right)
$$

and

$$
G_{N+M}\left(x_{1}^{\prime}, \cdots, x_{N}^{\prime} ; x_{1} \cdots x_{M}\right) \equiv\left\langle 0\left|T\left(\phi\left(x_{1}^{\prime}\right) \cdots \phi\left(x_{N}^{\prime}\right) \phi\left(x_{1}\right) \cdots \phi\left(x_{M}\right)\right)\right| 0\right\rangle
$$

It is now convenient to regard all the momenta as ingoing, relabel them as $q_{1}, q_{2}, \cdots q_{N+M}$ and write

$$
\left.\left\langle-q_{1}, q_{2}, \cdots-q_{N} ; \text { out }\right| q_{N+1}, \cdots q_{N+M} ; \text { in }\right\rangle=\int \mathcal{I} G_{N+M},
$$

where

$$
\mathcal{I}=\prod_{i=1}^{N+M} d^{4} x_{i} e^{-i q_{i} \cdot x_{i}} i\left(\partial_{i}^{2}+m^{2}\right)
$$

and

$$
G_{N+M}\left(x_{1}, \cdots, x_{N+M}\right)=\langle 0| T\left(\phi\left(x_{1}\right) \cdots \phi\left(x_{N+M}\right)|0\rangle .\right.
$$

We will later see the differences in the scattering amplitude on the GM plane through an analysis of the Gell-MannLow formula.

\section{B. The Gell-Mann-Low Formula}

The Heisenberg fields $\phi$ and the free fields $\phi_{F}$ at time $t=0$ fulfill the same canonical algebra if the interaction has no time derivatives. We assume that to be the case.

Then in perturbation theory, we choose the same representation of the canonical algebra at time 0 , namely that of the free field $\phi_{F}$ :

$$
\phi(\cdot, 0)=\phi_{F}(\cdot, 0)
$$

This implies that

$$
\begin{aligned}
\phi_{F}(\cdot, t) & =e^{i H_{0} t} \phi_{F}(\cdot, 0) e^{-i H_{0} t} \\
\phi(\cdot, t) & =e^{i H t} \phi(\cdot, 0) e^{-i H t}=e^{i H t} \phi_{F}(\cdot, 0) e^{-i H t}
\end{aligned}
$$


or

$$
\phi(\cdot, t)=\left(e^{i H t} e^{-i H_{0} t} \phi_{F}(\cdot, t)\left(e^{i H_{0} t} e^{-i H t}\right)\right.
$$

Let us define

$$
U\left(t_{1}, t_{2}\right)=e^{i t_{1} H_{0}} e^{-i\left(t_{1}-t_{2}\right) H} e^{-i t_{2} H_{0}}
$$

Then

$$
\begin{aligned}
U(t, t) & =1 \\
i \frac{\partial U}{\partial t_{1}}\left(t_{1}, t_{2}\right) & =H_{I}\left(t_{1}\right) U\left(t_{1}, t_{2}\right),
\end{aligned}
$$

where

$$
H_{I}(t)=e^{i t_{1} H_{0}} H_{I}(0) e^{-i t_{1} H_{0}}, \quad H_{I}(0)=H_{I}
$$

is the interaction representation Hamiltonian.

Thus

$$
U\left(t_{1}, t_{2}\right)=T \exp \left(-i \int_{t_{1}}^{t_{2}} d t H_{I}(t)\right)
$$

and

$$
\phi(\cdot, t)=U(0, t) \phi_{F}(\cdot, t) U(t, 0)
$$

Gell-Mann and Low show that

$$
G_{N+M}\left(x_{1}, \cdots x_{N+M}\right)=\frac{\left\langle 0, \mathrm{~F}\left|T\left(\phi_{F}\left(x_{1}\right) \cdots \phi_{F}\left(x_{N+M}\right) e^{i \int d^{4} x \mathcal{L}_{I}(x)}\right)\right| 0, \mathrm{~F}\right\rangle}{\left\langle 0, \mathrm{~F}\left|e^{i \int d^{4} x \mathcal{L}_{I}(x)}\right| 0, \mathrm{~F}\right\rangle}
$$

where $|0, \mathrm{~F}\rangle$ is the vacuum of the free Hamiltonian $H_{0}$ :

$$
H_{0}|0, \mathrm{~F}\rangle=0 \text {. }
$$

The proof is standard and will be omitted here.

\section{The twisted quantum fields}

Let us look at the case when $\theta^{\mu \nu} \neq 0$, first focussing on the situation with no gauge fields. Gauge fields will be treated later.

Our assumption is that the noncommutative field theory comes from the commutative one by the replacement

$$
\phi_{\theta}=\phi_{0} e^{\frac{1}{2} \overleftarrow{\partial} \wedge P}
$$

for matter fields, whereas gauge fields are not twisted (See also (27)). As $t \rightarrow \pm \infty$, the Heisenberg field $\phi_{0}$ for $\theta_{\mu \nu}=0$ becomes the corresponding in- and out- fields $\phi_{0}^{\text {in, out }}$. As for $P_{\mu}$, they are not affected by these limits, being constants of motion. Hence formally, we find, for the in- and out- fields $\phi_{\theta}^{\text {in,out }}$ of $\phi_{\theta}$, the results

$$
\phi_{\theta} \rightarrow \phi_{\theta}^{\text {in,out }}:=\phi_{\text {in }, \text { out }} e^{\frac{1}{2} \overleftarrow{\partial} \wedge P} \quad \text { as } \quad t \rightarrow \mp \infty
$$

For the twisted in and out annihilation and creation operators $a_{k}^{\text {in,out }}, a_{k}^{\dagger i n, o u t}$ we thus find

$$
a_{k}^{\text {in,out }}=c_{k}^{\text {in,out }} e^{-\frac{i}{2} k_{\mu} \theta^{\mu \nu} P_{\nu}}, \quad a_{k}^{\dagger i n, \text { out }}=c_{k}^{\dagger i n, \text { out }} e^{\frac{i}{2} k_{\mu} \theta^{\mu \nu} P_{\nu}} .
$$

There is a further convention we want to explain. For consistency with our notation for the coproduct on the Poincaré group[11], we define

$$
a_{k}^{\dagger i n}\left|k_{r}, k_{r-1}, \cdots, k_{1}\right\rangle_{\text {in }}=\left|k_{r}, k_{r-1}, \cdots, k_{1}, k\right\rangle_{\text {in }}
$$


and similarly for the action of $a_{k}^{\dagger o u t}$. Thus for example

$$
\left|k_{r}, k_{r-1}, \cdots, k_{1}\right\rangle_{\text {in }}=a_{k_{1}}^{\dagger i n} a_{k_{2}}^{\dagger i n} \cdots a_{k_{r}}^{\dagger i n}|0\rangle_{\text {in }} .
$$

and

$$
\left\langle-q_{N},-q_{N-1}, \cdots-q_{1} ; \text { out } \mid q_{N+M}, q_{N+M-1}, \cdots, q_{N+1}\right\rangle=\int \mathcal{I} G_{N+M}^{\theta}\left(x_{1}, x_{2}, \cdots, x_{N+M}\right)
$$

$G_{N+M} \equiv G_{N+M}^{0}$ has got changed to $G_{N+M}^{\theta}$ for $\theta_{\mu \nu} \neq 0$ where

$$
\begin{aligned}
G_{N+M}^{\theta}\left(x_{1}, \cdots x_{N+M}\right) & =T e^{\frac{i}{2} \sum_{I<J} \partial_{x_{I}} \wedge \partial_{x_{J}}} W_{N+M}^{0}\left(x_{1}, \cdots x_{N+M}\right) \\
: & =T W_{N+M}^{\theta}\left(x_{1}, \cdots x_{N+M}\right)
\end{aligned}
$$

and $W_{N+M}^{0}$ are the standard Wightman functions for untwisted fields:

$$
W_{N+M}^{0}\left(x_{1}, \cdots x_{N+M}\right)=\left\langle 0\left|\phi_{0}\left(x_{1}\right) \cdots \phi_{0}\left(x_{N+M}\right)\right| 0\right\rangle .
$$

It is important that because of translational invariance, the $W_{N+M}^{\theta}$ (and hence the $G_{N+M}^{\theta}$ ) depend only on coordinate differences.

For simplicity, we have included only matter fields, and that too of one type, in (70). Gauge fields can be included too, but they are not acted on by the twist exponential in (71).

The scattering matrix element is thus:

$$
\left.{ }_{\theta}\left\langle-q_{N},-q_{N-1}, \cdots-q_{1} ; \text { out }\right| q_{N+M}, q_{N+M-1}, \cdots, q_{N+1} ; \text { in }\right\rangle_{\theta}=\int \mathcal{I} G_{N+M}^{\theta}\left(x_{1}, x_{2}, \cdots, x_{M+N}\right)
$$

where $\mathcal{I}$ is as defined in (51).

On Fourier transforming as in (52), the $\theta_{i j}$ (space-space) part of the twist can be partially integrated. It gives the usual phase $e^{\frac{i}{2} q_{I}^{i} \theta_{i j} q_{J}^{j}}$. The time step-function (in the time-ordering $\mathrm{T}$ ) in (71) does not affect this manipulation.

To handle the $\theta_{0 i}$ part, consider a typical term

$$
\begin{aligned}
g_{N+M}^{\theta}\left(x_{1} \cdots x_{N+M}\right)= & \theta\left(x_{1}^{0}-x_{2}^{0}\right) \theta\left(x_{2}^{0}-x_{3}^{0}\right) \cdots \theta\left(x_{N+M-1}^{0}-x_{N+M}^{0}\right) \\
& e^{\frac{i}{2} \sum_{I<J} \partial_{x_{I}} \wedge \partial_{x_{J}}} W_{N+M}^{0}\left(x_{1}, \cdots x_{N+M}\right) .
\end{aligned}
$$

which occurs on expanding the time-ordered product in terms of retarded products.

The twist here is the product of terms

$$
e^{\frac{i}{2}\left[\partial_{x_{I}^{0}} \theta \cdot \nabla_{J}-\left(\theta \cdot \nabla_{I}\right) \partial_{x_{J}^{0}}\right]}, \quad I<J, \quad \theta \cdot \nabla_{J} \equiv \theta_{0 i} \partial_{x_{J}^{i}}
$$

on retaining just $\theta_{0 i}$. The coefficient of $\partial_{x_{I}^{0}}$ in the exponential is thus

$$
\sum_{J>I} \theta \cdot \nabla_{J}-\sum_{J<I} \theta \cdot \nabla_{J}
$$

On partial integration in eq(73) $\nabla_{J}$ becomes $i q_{J}$ and

$$
e^{\frac{i}{2} \partial_{x_{I}^{0}}\left(\sum_{J>I}-\sum_{J<I}\right) \theta \cdot \nabla_{J}} \rightarrow e^{-\frac{1}{2}\left(\sum_{J>I} \theta \cdot q_{J}-\sum_{J<I} \theta \cdot q_{J}\right) \partial_{x_{I}^{0}}}
$$

This translates the $x_{I}^{0}$ 's according to

$$
\begin{aligned}
x_{I-1}^{0} & \rightarrow x_{I-1}^{0}-\frac{1}{2}\left(\sum_{J>I-1} \theta \cdot q_{J}-\sum_{J<I-1} \theta \cdot q_{J}\right), \\
x_{I}^{0} & \rightarrow x_{I}^{0}-\frac{1}{2}\left(\sum_{J>I} \theta \cdot q_{J}-\sum_{J<I} \theta \cdot q_{J}\right), \\
x_{I+1}^{0} & \rightarrow x_{I+1}^{0}-\frac{1}{2}\left(\sum_{J>I+1} \theta \cdot q_{J}-\sum_{J<I+1} \theta \cdot q_{J}\right)
\end{aligned}
$$


Or

$$
x_{I-1}^{0}-x_{I}^{0} \rightarrow\left(x_{I-1}^{0}-x_{I}^{0}\right)-\frac{1}{2} \theta \cdot q_{J}+\frac{1}{2} \sum_{J \neq I-1, I} \theta \cdot q_{J} .
$$

But $\sum \vec{q}_{J}=0$, so that

$$
x_{I-1}^{0}-x_{I}^{0} \rightarrow x_{I-1}^{0}-x_{I}^{0}-\frac{1}{2} \theta \cdot q_{I-1}-\frac{1}{2} \theta \cdot q_{I}
$$

Similarly,

$$
x_{I}^{0}-x_{I+1}^{0} \rightarrow x_{I}^{0}-x_{I+1}^{0}-\frac{1}{2} \theta \cdot q_{I}-\frac{1}{2} \theta \cdot q_{I+1}
$$

From (82/83), we see that each $x_{I}^{0}$ is (time) shifted to

$$
x_{I}^{0}+\delta x_{I}^{0}, \quad \delta x_{I}^{0}=\delta x_{I}^{0}\left(q_{1}, \cdots q_{N}\right) .
$$

where the $\delta x_{I}^{0}$ actually depend on the ordering on $x_{I}^{0}$. No further simplification seems possible.

We emphasize the following important observations.

- Firstly, (73) involves only the $\theta^{\mu \nu}=0$ fields in $W_{N}^{0}$. So it can be used to map any commutative theory to noncommutative one, including also the standard model. But special care is needed to treat gauge fields. Gauge fields are not twisted unlike matter fields. As explained elselwhere, this means that the Yang-Mills tensor is not twisted, $F_{\theta}^{\mu \nu}=F_{0}^{\mu \nu}$. But covariant derivatives of matter fields $\phi_{\theta}$ are twisted: $\left(D_{\mu} \phi\right)_{\theta}=\left(D_{\mu} \phi\right)_{0} e^{\frac{1}{2} \overleftarrow{\partial} \wedge P}$. where $\left(D_{\mu} \phi\right)_{0}$ is the untwisted covariant derivative of the untwisted $\phi_{0}$. Thus in correlators $W_{N}^{\theta}$, we must use $\left(D_{\mu} \phi\right)_{\theta}$ for matter fields, $F_{0}^{\mu \nu}$ for Yang-Mills tensor.

- There are ambiguities in formulating scattering theory. Thus if we substitute (66) directly in the LHS of (52), we get our earlier result [1]. This corresponds to putting the twist factor in (75) outside the symbol $\mathrm{T}$ in (71). At this moment, lacking a rigorous scattering theory, we do not know which of these is the correct answer. In this connection, we must mention the important work of Buchholz and Summers [12] which rigorously develops the wedge localisation ideas of Grosse and Lechner to establish a scattering theory for two incoming and two outgoing particles. The result resembles those in our earlier approach [8], but there seem to be descrepencies in the signs of the momenta in the overall phases.

For calculating (73), we need a formalism for doing perturbation theory to calculate Wightman functions. Once we have that, we can calculate the time-ordered product by writing it in terms of Wightman functions and twist factors. We show such a calculation below.

\section{Perturbation Theory for Wightman Functions}

Perturbation theory for Wightman functions is available [13]. We can also construct this formalism directly. For free fields, Wightman functions are Gaussian correlated. For example,

$$
\left\langle\phi_{F}\left(x_{1}\right) \phi_{F}\left(x_{2}\right) \phi_{F}\left(x_{3}\right) \phi_{F}\left(x_{4}\right)\right\rangle=\left\langle\phi_{F}\left(x_{1}\right) \phi_{F}\left(x_{2}\right)\right\rangle\left\langle\phi_{F}\left(x_{3}\right) \phi_{F}\left(x_{4}\right)\right\rangle+\text { permutations }
$$

while for Dirac fields, there are signs attached to the succesive terms reflecting the signature of the permutations in $x_{1}, x_{2}, x_{3}, x_{4}$. The two-point functions here are well-known. For example,

$$
\left\langle\phi_{F}\left(x_{1}\right) \phi_{F}\left(x_{2}\right)\right\rangle=\Delta_{+}\left(x_{1}-x_{2}, m^{2}\right) .
$$

Now to calculate Wightman functions for interacting fields, we can expand Heisenberg fields $\phi$ in terms of free fields using (62) and express the resultant free field correlators in terms of two point functions.

As an illustration, consider

$$
\begin{aligned}
& \left\langle T\left(\phi_{F}\left(x_{1}\right) \phi_{F}\left(x_{2}\right) \phi_{F}\left(x_{3}\right) \phi_{F}\left(x_{4}\right)\right)\right\rangle= \\
& \quad \theta\left(x_{1}^{0}-x_{2}^{0}\right) \theta\left(x_{2}^{0}-x_{3}^{0}\right) \theta\left(x_{3}^{0}-x_{4}^{0}\right)\left\langle\phi_{F}\left(x_{1}\right) \phi_{F}\left(x_{2}\right) \phi_{F}\left(x_{3}\right) \phi_{F}\left(x_{4}\right)\right\rangle+\cdots
\end{aligned}
$$

The Wightman function is then given by (85). We can similarly calculate the remaining terms in (87).

Renormalisation theory for Wightman functions has also been developed [13]. 


\section{SUMMARY OF OUR RULES FOR TWISTED QUANTUM FIELD THEORIES}

Our rules for transition from the $\theta^{\mu \nu}=0$ to the $\theta^{\mu \nu} \neq 0$ theory are simple and definite. Let us focus on scattering amplitudes. They are given by reduction formulae as in (73). They show that to compute scattering amplitudes for $\theta^{\mu \nu} \neq 0$, we need a formula for twisted Wighman functions $W_{N+M}^{\theta}$ in (71) in terms of the untwisted Wightman

functions $W_{N+M}^{0}$. We have already explained this formula: the passage from $W_{N+M}^{0}$ to $W_{N+M}^{\theta}$ is achieved by twisting all fields except the gauge fields for unbroken gauge symmetries $H$. Further if $D(V)^{\theta=0}$ is the connection with gauge potential $V$ for the unbroken group $H$, then the covariant derivative for a twisted matter field $\psi^{\theta}$ is to be defined as $D_{\mu}^{\theta}(V) \psi^{\theta}:=\left(D_{\mu}(V)^{0} \psi^{0}\right) e^{\frac{1}{2} \overleftarrow{\partial} \wedge P}$.

This rule preserves the asymptotic conditions and shows that the spectrum of the theory is unaltered by changing $\theta^{\mu \nu}$. It covers theories with spontaneous symmetry breakdown as well provided we have a scheme for treating it for $\theta^{\mu \nu}=0$. We remark in this connection that since twist factors with time derivatives occur in (71) within the timeordering symbol and the amount of time-translation they generate depend on external momenta, the Gell-Mann-Low formula has to be modified significantly.

\section{SPONTANEOUS SYMMETRY BREAKING}

This topic is of sufficient importance that we discuss it separately. The consistency of this discussion with what we discussed earlier will be apparent.

Let us first consider the case of spontaneously broken global symmetries. Suppose we have a multiplet of quantum fields $\phi_{i}(x)$ that transforms under the action of (some representation $D(g)$ of) a symmetry group $G$ according to

$$
\phi_{i}(x) \rightarrow \phi_{i}^{g}(x)=D(g)_{i j} \phi_{j}(x)
$$

If this is a symmetry of the theory, then the quantum charges $Q_{0}^{a}$ commute with the full Hamiltonian:

$$
\left[Q_{0}^{a}, H\right]=0, \quad a=1,2, \cdots \operatorname{dim} G .
$$

These conventionally arise from quantum currents $J^{a, \mu}(x)$ which are conserved:

$$
\partial_{\mu} J_{0}^{a, \mu}=0
$$

where the currents $J_{0}^{a, \mu}$ are constructed from the quantum fields $\phi_{i, 0}(x)$ and its derivatives.

Given a commutative quantum theory with conserved currents $J_{0}^{a, \mu}$, it is easy to see that in the corresponding noncommutative theory (obtained by replacing $\phi_{i, 0}(x) \rightarrow \phi_{i, \theta}(x)=\phi_{i, 0}(x) e^{\frac{1}{2} \overleftarrow{\partial} \wedge P}$ ), the noncommutative currents $J_{\theta}^{a, \mu}(x)=J_{0}^{a, \mu}(x) e^{\frac{1}{2} \overleftarrow{\partial} \wedge P}$ are also conserved:

$$
\partial_{\mu} J_{\theta}^{a, \mu}(x)=\partial_{\mu} J_{0}^{a, \mu}(x) e^{\frac{1}{2} \overleftarrow{\partial} \wedge P}=\left(\partial_{\mu} J_{0}^{a, \mu}(x)\right) e^{\frac{1}{2} \overleftarrow{\partial} \wedge P}=0
$$

Interestingly, the charges $Q_{\theta}^{a}$ in the noncommutative theory

$$
Q_{\theta}^{a}=\int d^{3} x J_{\theta}^{a, 0}(x)=\int d^{3} x J_{0}^{a, 0}(x) e^{\frac{1}{2} \overleftarrow{\partial} \wedge P}=\int d^{3} x J_{0}^{a, 0}(x)=Q_{0}^{a}
$$

are the same as in the commutative case. The last equation follows using integration by parts for terms involving $\theta_{i j}$ and using the time independence of the charges of the commutative theory for the rest. The charges $Q_{0}^{a}, Q_{\theta}^{a}$ generate the infinitesimal symmetry transformations:

$$
\begin{aligned}
{\left[Q_{0}^{a}, \phi_{i, 0}(x)\right] } & =\sum_{j} T_{i j}^{a} \phi_{j, 0}(x), \\
{\left[Q_{\theta}^{a}, \phi_{i, \theta}(x)\right] } & =\sum_{j} T_{i j}^{a} \phi_{j, \theta}(x) .
\end{aligned}
$$

\section{A. Goldstone's theorem}

Consider the vacuum expectation value of the commutator of the currents $J_{\theta}^{a, \mu}(y)$ and the quantum field $\phi_{i, \theta}(x)$ :

$$
\left\langle 0\left|\left[J_{\theta}^{a, \mu}(y), \phi_{i, \theta}(x)\right]\right| 0\right\rangle=\left\langle 0\left|\left[e^{\frac{1}{2} \overrightarrow{\partial_{y}} \wedge P} J_{0}^{a, \mu}(y), \phi_{0}(x) e^{\frac{1}{2} \overleftarrow{\partial_{x}} \wedge P}\right]\right| 0\right\rangle .
$$


The first term in the commutator here is

$$
e^{\frac{i}{2} \partial_{y} \wedge \theta \partial_{x}}\left\langle 0\left|J_{0}^{a, \mu}(y) \phi_{0}(x)\right| 0\right\rangle=e^{\frac{i}{2} \partial_{y} \wedge \theta \partial_{x}}\left\langle 0\left|J_{0}^{a, \mu}(0) \phi_{0}(x-y)\right| 0\right\rangle
$$

where we have used translational invariance and the fact that

$$
\partial_{y} \wedge \partial_{x}\left\langle 0\left|J_{0}^{\mu}(0) \phi_{0}(x-y)\right| 0\right\rangle=-\partial_{x} \wedge \partial_{x}\left\langle 0\left|J_{0}^{\mu}(0) \phi_{0}(x-y)\right| 0\right\rangle=0
$$

in the second step. The $\theta^{\mu \nu}$ dependence in the second term as well disappears in the same manner so that

$$
\left\langle 0\left|\left[J_{\theta}^{a, \mu}(y), \phi_{i, \theta}(x)\right]\right| 0\right\rangle=\left\langle 0\left|\left[J_{0}^{a, \mu}(y), \phi_{i, 0}(x)\right]\right| 0\right\rangle .
$$

This commutator being the same as the one for the corresponding commutative case, the standard arguments using spectral density and Lorentz invariance [14] can be used to argue for the existence of massless bosons in the symmetrybroken phase. Following [15], we reproduce this argument below.

Summing over intermediate states, and using Lorentz invariance, the vacuum expectation value of the commutator may be expressed as

$$
\left\langle 0\left|\left[J_{0}^{a, \mu}(y), \phi_{i, 0}(x)\right]\right| 0\right\rangle=\int d^{4} p\left(\rho_{i}^{a, \mu}(p) e^{-i p \cdot(y-x)}-\tilde{\rho}_{i}^{a, \mu}(p) e^{i p \cdot(y-x)}\right)
$$

where the spectral densities $\rho_{i}^{a}(p), \tilde{\rho}_{i}^{a}(p)$ are defined as

$$
\begin{aligned}
& \rho_{i}^{a, \mu}(p)=\sum_{N}\left\langle 0\left|J_{0}^{a, \mu}(0)\right| N\right\rangle\left\langle N\left|\phi_{i, 0}(0)\right| 0\right\rangle \delta^{4}\left(p-p_{N}\right), \\
& \tilde{\rho}_{i}^{a, \mu}(p)=\sum_{N}\left\langle 0\left|\phi_{i, 0}(0)\right| N\right\rangle\left\langle N\left|J_{0}^{a, \mu}(0)\right| 0\right\rangle \delta^{4}\left(p-p_{N}\right),
\end{aligned}
$$

and $p_{N}$ is the total four-momentum in the state $|N\rangle$.

By Lorentz invariance and non-negativity of energy, these densities are of the form

$$
\begin{aligned}
& \rho_{i}^{a, \mu}(p)=p^{\mu} \rho_{i}^{a}\left(p^{2}\right) \theta\left(p^{0}\right), \\
& \tilde{\rho}_{i}^{a, \mu}(p)=p^{\mu} \tilde{\rho}_{i}^{a}\left(p^{2}\right) \theta\left(p^{0}\right),
\end{aligned}
$$

which implies that

$$
\begin{aligned}
& \left\langle 0\left|\left[J_{0}^{a, \mu}(y), \phi_{i, 0}(x)\right]\right| 0\right\rangle= \\
& \quad i \frac{\partial}{\partial y_{\mu}} \int d M^{2}\left(\rho_{i}^{a}\left(M^{2}\right) \Delta_{+}\left(y-x ; M^{2}\right)+\tilde{\rho}_{i}^{a}\left(M^{2}\right) \Delta_{+}\left(x-y ; M^{2}\right)\right)
\end{aligned}
$$

where $\Delta_{+}\left(x ; M^{2}\right)$ is the standard two-point Wightman function:

$$
\Delta_{+}\left(x ; M^{2}\right)=\int d \mu(p) e^{-i p \cdot x}, \quad \text { where } \quad d \mu(p)=\frac{d^{3} p}{2 p_{0}}, \quad p_{0}=\sqrt{\vec{p}^{2}+M^{2}}
$$

Since $\Delta_{+}\left(x ; M^{2}\right)$ and $\Delta_{+}\left(-x ; M^{2}\right)$ are equal for $x$ spacelike, we can write, for such $x-y$,

$$
\left\langle 0\left|\left[J_{0}^{a, \mu}(y), \phi_{i, 0}(x)\right]\right| 0\right\rangle=i \frac{\partial}{\partial y_{\mu}} \int d M^{2}\left(\rho_{i}^{a}\left(M^{2}\right)+\tilde{\rho}_{i}^{a}\left(M^{2}\right)\right) \Delta_{+}\left(y-x ; M^{2}\right) .
$$

For spacelike separations, the commutator vanishes, so that

$$
\rho_{i}^{a}\left(M^{2}\right)=-\tilde{\rho}_{i}^{a}\left(M^{2}\right)
$$

which gives us

$$
\left\langle 0\left|\left[J_{0}^{a, \mu}(y), \phi_{i, 0}(x)\right]\right| 0\right\rangle=i \frac{\partial}{\partial y_{\mu}} \int d M^{2} \rho_{i}^{a}\left(M^{2}\right)\left(\Delta_{+}\left(y-x ; M^{2}\right)-\Delta_{+}\left(x-y ; M^{2}\right)\right)
$$

Now, since the current $J_{0}^{a, \mu}$ is conserved, we can act by $\partial / \partial y_{\mu}$ to get

$$
0=\int d M^{2} M^{2} \rho_{i}^{a}\left(M^{2}\right)\left(\Delta_{+}\left(y-x ; M^{2}\right)-\Delta_{+}\left(x-y ; M^{2}\right)\right)
$$


and thus we get

$$
M^{2} \rho_{i}^{a}\left(M^{2}\right)=0
$$

Now consider the situation when the symmetry is broken. For $\mu=0, x^{0}=y^{0}=t$,

$$
\left\langle 0\left|\left[J_{0}^{a, 0}(\vec{y}, t), \phi_{i, 0}(\vec{x}, t)\right]\right| 0\right\rangle=i \delta(\vec{y}-\vec{x}) \int d M^{2} \rho_{i}^{a}\left(M^{2}\right) .
$$

Integrating and using (93), we get

$$
\sum_{j} T_{i j}^{a}<\phi_{j, 0}(x)>=i \int \rho_{i}^{a}\left(M^{2}\right)
$$

Eqs. (110) and (112) are compatible only if

$$
\rho_{i}^{a}\left(M^{2}\right)=i \delta\left(M^{2}\right) \sum_{j} T_{i j}^{a}\left\langle 0\left|\phi_{j, 0}(0)\right| 0\right\rangle
$$

As long as the symmetry is broken, the spectral density $\rho_{i}^{a}$ is proportional to $\delta\left(M^{2}\right)$. Since such a term can arise only in a theory with massless particles, we are forced to conclude that a broken symmetry with $T_{i j}^{a}\left\langle 0\left|\phi_{j, \theta}(0)\right| 0\right\rangle \neq 0$ requires the existence of a massless particle with the same quantum numbers as $J_{\theta}^{a, 0}$. These are nothing but the Goldstone bosons.

\section{B. Spontaneously Broken Local Symmetries \& twisted standard model}

Now given the map between the twisted fields and untwisted ones (eq. 65) and our earlier established rules for getting the correlation functions for the case of $\theta_{\mu \nu} \neq 0$ eqs. (71) and (74) we can easily see that the Higgs mechanism will follow with the mass of the gauge boson being identical to the untwisted case. We can readily understand this result from the fact that the in and out fields completely determine the mass spectrum and they remain independent of $\theta_{\mu \nu}$ because of formulae like (66) and (67).

The Hamiltonian $P_{0}=H$ and the spatial translation generator for the twisted standard model are the same as for the case $\theta^{\mu \nu}=0$ What is changed in our LSZ approach are the in and out fields which are twisted as discussed. Hence scattering calculations can be based on appropriately modified Wightman functions as we have already explained.

We will elsewhere calculate specific twisted standard model cross-sections and examine the new features coming from non-commutativity.

\section{FINAL REMARKS}

In this paper, we have outlined an approach for calculating the scattering amplitudes in twisted qft's from untwisted ones using LSZ formalism. It works in gauge theories with or without spontaneous breakdown. Implications for the standard model will be presented in a forthcoming paper.

As remarked earlier, the results for scattering matrix in this approach differs from the interaction representation perturbation theory. The reasons for this difference remain to be pinpointed.

In our judgement, since the LSZ approach works with fully interacting fields and total momentum $P_{\mu}$ (including also interactions), it is probably superior to the results based on interaction representation perturbation theory. It does not change $P_{\mu}$ in the process of twisting, but changes just the in- and out- fields appropriately to account for the twisted statistics. This change is forced on us when the coproduct of the Poincaré-Hopf algebra is twisted.

In the presence of matter and gauge fields, the coproduct for the Poincaré algebra becomes non-associative and gives rise to a Poincaré - quasi Hopf algebra [16]. We will discuss this quasi- Hopf algebra in detail in another paper.

Acknowledgments: The work of APB is supported in part by US-DOE under grant number DE-FG02-85ER40231 and the Universidad Carlos III de Madrid. The work of APB and TRG are supported by the DST CP-STIO program. 
[1] S. S. Gubser and S. L. Sondhi, Nucl.Phys. B 605, 395 (2001)

[2] W. Bietenholz, F. Hofheinz, J. Nishimura, Nucl. Phys B 119 Proc. Suppl. 941, (2003) hep-lat/0209021, J. Ambjorn and S. Catterall, Phys. Lett. B 549, 253 (2002); W. Bietenholz, F. Hofheinz, J. Nishimura, JHEP 0406 042 (2004); arXiv:hep-th/0404020. W. Bietenholz, J. Nishimura, Y. Susaki, J. Volkholz, JHEP 0610 042 (2006); arXiv:hep-th/0608072.

[3] C. R. Das, S. Digal and T. R. Govindarajan, Mod. Phys. Lett. A 23, 1781 (2008)

[4] C. R. Das, S. Digal and T. R. Govindarajan, arXiv:0801.4479 [Mod Phys Letts (to be published)]

[5] S. Minwalla, M. V. Raamsdonk and N. Seiberg, JHEP 0002020 (2000); arXiv:hep-th/9912072. A. P. Balachandran, A. Pinzul, A. R. Queiroz, Phys. Lett. B 668241 (2008); arXiv:0804.3588 [hep-th]].

[6] A.P. Balachandran, G. Mangano, A. Pinzul, S. Vaidya, Int. J. Mod. Phys. A 21, 3111 (2006); arXiv:hep-th/0508002.

[7] A. P. Balachandran, T. R. Govindarajan, G. Mangano, A. Pinzul, B. A. Qureshi and S. Vaidya, Phys. Rev. D 75, 045009 (2007); arXiv:hep-th/0608179.

[8] A. P. Balachandran, A. Pinzul and B. Qureshi, Phys. Lett. B 634, 434 (2006); arXiv:hep-th/0508151.

[9] A. P. Balachandran, A. Pinzul, B. Qureshi and S. Vaidya, Phys. Rev. D 76, 105025 (2007); arXiv:0708.0069 [hep-th]].

[10] H. Grosse, Phys. Lett. B 86, 267 (1979); A. B. Zamolodchikov and Al. B. Zamolodchikov, Annals Phys. 120, 253 (1979); L. Faddeev, Sov. Sci. Rev. C 1, 107 (1980).

[11] A. P. Balachandran, A. Pinzul, B. A. Qureshi and S. Vaidya, Phys. Rev. D 77025020 (2008); arXiv:0708.1379 [hep-th]]; A. P. Balachandran, A. Pinzul and B. A. Qureshi,Phys. Rev. D 77025021 (2008); arXiv:0708.1779 [hep-th]].

[12] D Buchholz and S J Summers, arXiv:0806.0349

[13] A. Ostendorf, A, Annales de l'institut Henri Poincaré (A) Physique théorique, 40, 273 (1984); O. Steinmann, Annales de l'institut Henri Poincaré (A) Physique théorique, 63, 399 (1995).

[14] J. Goldstone, A. Salam and S. Weinberg, Phys. Rev 127, 965 (1962).

[15] S. Weinberg, Quantum Theory of Fields II, Chap 19 (Cambridge University Press, Cambridge, England, 2000).

[16] A P Balachandran and B A Qureshi, [arXiv: 0903.0478/hep-th]. 